\title{
对甲苯硫酚/三环己基膦介导的硝基芳烃的光化学还原反应
}

\author{
鲍兆伟 吕洁金智超* \\ (贵州大学绿色农药与农业生物工程国家重点实验室培育基地 \\ 教育部绿色农药与农业生物工程重点实验室＼cjkstart贵阳 550025)
}

\begin{abstract}
摘要 报道了一种无溶剂、无金属温和条件下，对甲苯硫酚/三环己基膦介导的硝基芳烃的光化学还原反应. 该反应具 有广泛的普遍适用性，带有不同取代基和取代模式的胺类衍生物可以获得良好的产率. 因此该还原方法是一种具有潜 在价值的高效合成胺类化合物的新策略.
\end{abstract}

关键词 硝基芳烃; 还原反应; 光化学; 芳香胺

\section{Photochemical Reduction of Nitroaromatics Mediated by p-Toluenethiol/PCy}

\author{
Bao, Zhaowei Lü, Jie Jin, Zhichao* \\ (State Key Laboratory Breeding Base of Green Pesticide and Agricultural Bioengineering, Key Laboratory of Green \\ Pesticide and Agricultural Bioengineering, Ministry of Education, Guizhou University, Guiyang 550025)
}

\begin{abstract}
A new photochemical reduction of nitroaromatics mediated by $p$-toluenethiol and PCyз under solvent-free, metal-free, and mild conditions is reported for the first time. This reaction has shown good generalities, and amine derivatives with different substituents and substitution patterns can be obtained in good yields. Therefore, this reduction method represents a potentially valuable new strategy for the efficient synthesis of amine compounds.

Keywords nitroaromatics; reduction; photochemistry; aromatic amines
\end{abstract}

胺类衍生物在农药 ${ }^{[1]}$ 、医药 ${ }^{[2]}$ 和天然产物 ${ }^{[3]}$ 中是一类 常见的化合物, 同时胺类化合物很容易进行下一步功能 性衍生化, 这让胺类化合物成为合成各种精细化学品的 重要中间体 ${ }^{[4]}$, 广泛应用于医药、农药及颜料等相关领 域. 由于胺类化合物具有广泛的实用性, 因此开发合成 胺类化合物的新型方法是一个活跃的研究领域. 目前胺 类化合物的合成方法有很多种, 如还原硝基、还原亚胺 和还原氰基等, 其中还原硝基化合物是工业中使用最为 广泛的方法. 传统的硝基还原方法需要使用 $\mathrm{Pd} / \mathrm{C} 、 \mathrm{Pt} / \mathrm{C}$ 、 $\mathrm{Fe} / \mathrm{HCl} 、$ Raney Ni、Co、Ru 和 Rh 等金属催化剂 ${ }^{[5]}$ (Scheme 1a), 因此存在一些弊端, 比如 $\mathrm{Fe} / \mathrm{HCl}$ 还原硝基会产生 大量的铁泥废渣, 对环境造成严重污染. 过渡金属催化 氢化的催化剂( $\mathrm{Pd} 、 \mathrm{Pt} 、 \mathrm{Ni} 、 \mathrm{Ru}$ 和 $\mathrm{Rh}$ 等)价格昂贵, 底 物适用范围有限, 可操作性差, 反应条件也较为苛刻 (高温、高压氢气). 因此为该类还原反应开发出新型的
还原方法是一项非常有意义的研究. 近年来很多课题组 陆续报道了新型、有效、实用的还原方法 ${ }^{[6]}$, 这为进一 步开发硝基化合物的还原方法提供了有价值的参考. 比 如 Benaglia 课题组 ${ }^{[7]}$ 开发了使用 $\mathrm{HSiCl}_{3} / \mathrm{DIPEA}$ (二异丙 基乙基胺)作为还原条件，以 $\mathrm{MeCN}$ 作为溶剂将烷基类 硝基化合物还原成氰基化合物或者胺类化合物，该方法 表现出了优异的官能团耐受性, 并且不会影响分子的手 性中心. 与此同时, Oestreich 课题组 ${ }^{[8]}$ 在 $100{ }^{\circ} \mathrm{C}$ 下, 使 用 $\mathrm{B}\left(\mathrm{C}_{6} \mathrm{~F}_{5}\right)_{3} / \mathrm{Et}_{3} \mathrm{SiH}$ 分别作为还原剂和氢源供体来还原 硝基化合物，得到胺类化合物，并获得了良好的收率. 在这些研究中, 叔膦用于还原硝基化合物的研究较少. 2005 年, Freeman 课题组 [9]利用三苯基膦还原 2-硝基联 苯衍生物, 合成了一系列氮杂-吡啶稠合杂芳烃衍生物 (Scheme 1b). 2010 年, 刘小钢课题组 ${ }^{[10]}$ 发现了一个新型 的氢原子供体，报道了一种用硫醇或者硫酚来还原卤素

\footnotetext{
* Corresponding author. E-mail: zcjin@gzu.edu.cn

Received September 24, 2021; revised October 26, 2021; published online November 3, 2021.

Project supported by the National Natural Science Foundation of China (Nos. 21801051, 21961006, 32172459), and the Science and Technology Department of Guizhou Province (No. Qiankehejichu-ZK[2021]Key033).

国家自然科学基金(Nos. 21801051, 21961006, 32172459)及贵州省科技技术基金(No. 黔科合基础-ZK[2021]重点 033)资助项目.
} 
取代的芳香烃硝基化合物, 得到苯硫醚取代的胺类衍生 物(Scheme 1c). 可见光诱导的有机小分子转化反应具有 条件温和、选择性高和可控性好等优点 ${ }^{[11]}$. 最近基于可 见光诱导, 光化学工作者进行了一系列有意义的研究工 作 ${ }^{[12]}$, 尤其是魏伟课题组 ${ }^{[13]}$ 和何卫民课题组 ${ }^{[11,14]}$, 报道 了很多反应条件温和、实验操作简便、安全性好、环境 友好的反应模式, 这对光化学反应的发展具有重要的启 示意义. 2021 年, 黄华文课题组 ${ }^{[15]}$ 报道了在光照条件下, 利用协同的 $\mathrm{NaI} / \mathrm{PPh}_{3}$ 催化体系与 $\mathrm{PhSiH}_{3}$ 作为氢原子供 体进行还原加氢, 得到了胺类化合物(Scheme 1d). 基于 以上相关的研究, 我们设计使用叔膦作为还原剂, 苯硫 酚作为氢源供体, 在可见光诱导下将硝基芳烃类化合物 还原为胺类化合物 (Scheme 1e).

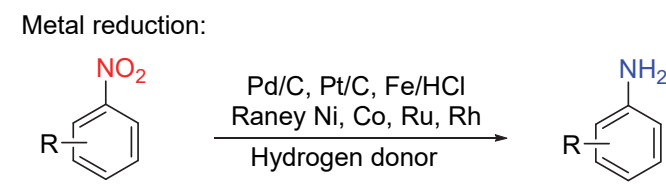

Metal-free reduction:

Freeman (2005): $\mathrm{PR}_{3}$ as a reducing agent

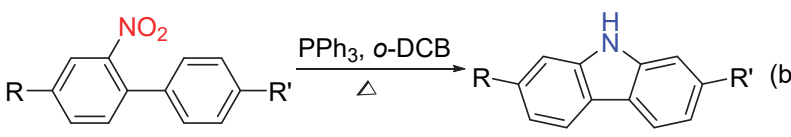

Liu (2010): RSH as a hydrogen source donor

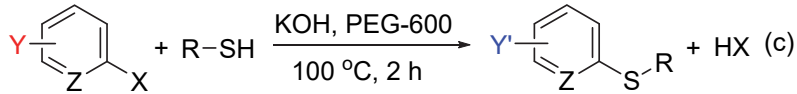

$$
\begin{aligned}
& X=\mathrm{F}, \mathrm{Cl} ; \mathrm{Y}=\mathrm{NO}_{2}
\end{aligned}
$$

Huang (2021): Photochemical reduction

$$
\text { Ar, Blue LEDs }
$$

This work:

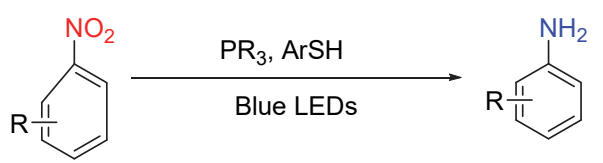

图式 1 硝基芳烃类化合物的还原反应

Scheme 1 Reduction of nitroaromatic compounds

\section{1 结果与讨论}

以简单易得且稳定的对硝基联苯(1a)作为模型底 物, 对反应进行条件优化, 关键结果如表 1 所示. 在对 甲基苯硫酚 (A) 作为氢源供体, 水作为溶剂的条件下, 首先尝试不同种类的叔膦进行反应(表 1, Entries 1 4). 根据反应结果显示, 当三环己基膦 $\left(\mathbf{P}_{3}\right)$ 作为还原剂时, 目标产物 $\mathbf{2 a}$ 的产率为 $75 \%$. 接着以三环己基膦 $\left(\mathbf{P}_{3}\right)$ 作为
还原剂考察溶剂对反应的影响，遗憾的是产物 2a 的产 率并没有提高(表 1, Entries 5,6). 随后又考察了不同氢 源供体对反应产率的影响，结果显示其它的氢源供体会 导致产物 2a 的产率降低, 甚至观察不到目标产物 $2 \mathrm{a}$ (表 1, Entries 7 9). 为了得到更好的产率, 尝试进一步提 高对甲基苯硫酚 $(\mathbf{A})$ 和三环己基膦 $\left(\mathbf{P}_{3}\right)$ 的用量, 并缩短反 应时间为 $3 \mathrm{~h}$, 结果发现使用 $300 \mathrm{~mol} \%$ 的三环已基膦和 对甲基苯硫酚时，目标产物 $\mathbf{2 a}$ 可以达到 93\%的产率(表 1, Entry 10). 最后, 我们惊奇地发现，在不加任何溶剂 的情况下，目标产物 2a 的产率可以达到 98\%(表 1, Entry 11).

表 1 硝基芳烃 $1 \mathrm{a}$ 的还原反应条件优化 ${ }^{a}$

Table 1 Condition optimization for the reduction of the nitroarene 1a
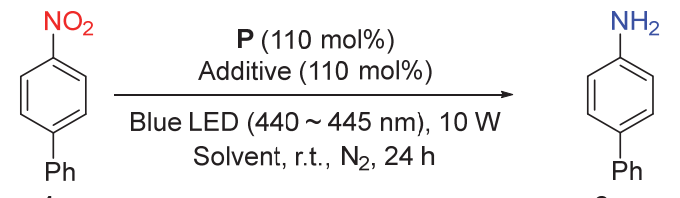

2a

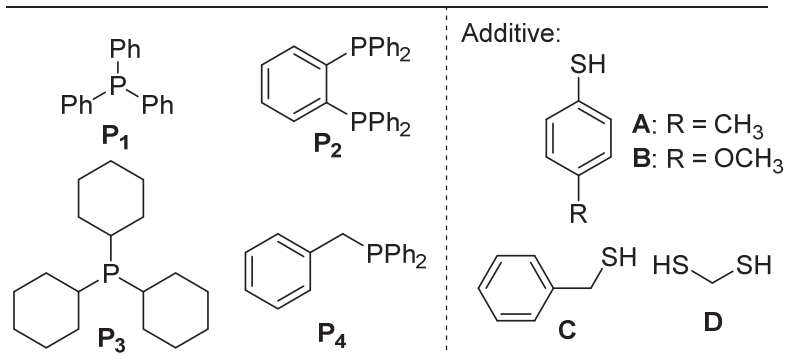

\begin{tabular}{ccccc}
\hline Entry & $\mathbf{P}$ & Solvent & Additive & Yield $^{b} / \%$ \\
\hline 1 & $\mathbf{P}_{\mathbf{1}}$ & $\mathrm{H}_{2} \mathrm{O}$ & $\mathbf{A}$ & 67 \\
2 & $\mathbf{P}_{\mathbf{2}}$ & $\mathrm{H}_{2} \mathrm{O}$ & $\mathbf{A}$ & 50 \\
3 & $\mathbf{P}_{\mathbf{3}}$ & $\mathrm{H}_{2} \mathrm{O}$ & $\mathbf{A}$ & 75 \\
4 & $\mathbf{P}_{\mathbf{4}}$ & $\mathrm{H}_{2} \mathrm{O}$ & $\mathbf{A}$ & 66 \\
5 & $\mathbf{P}_{\mathbf{3}}$ & $\mathrm{MeOH}$ & $\mathbf{A}$ & 55 \\
6 & $\mathbf{P}_{\mathbf{3}}$ & $i-\mathrm{PrOH}$ & $\mathbf{A}$ & 13 \\
7 & $\mathbf{P}_{3}$ & $\mathrm{H}_{2} \mathrm{O}$ & $\mathbf{B}$ & 69 \\
8 & $\mathbf{P}_{3}$ & $\mathrm{H}_{2} \mathrm{O}$ & $\mathbf{C}$ & 0 \\
9 & $\mathbf{P}_{3}$ & $\mathrm{H}_{2} \mathrm{O}$ & $\mathbf{D}$ & 0 \\
$10^{c}$ & $\mathbf{P}_{\mathbf{3}}$ & $\mathrm{H}_{2} \mathrm{O}$ & $\mathbf{A}$ & 93 \\
$11^{c}$ & $\mathbf{P}_{\mathbf{3}}$ & - & $\mathbf{A}$ & 98 \\
\hline
\end{tabular}

${ }^{a}$ General conditions: 1a $(0.10 \mathrm{mmol}), \mathbf{P}(0.11 \mathrm{mmol})$, additive $(0.11 \mathrm{mmol})$, blue LED $440 \sim 445 \mathrm{~nm}, 10 \mathrm{~W}$ and solvent $(2.0 \mathrm{~mL})$ at r.t. for 24 h. ${ }^{b}$ Isolated yield of 2a. ${ }^{c}$ With $0.30 \mathrm{mmol}$ of $\mathbf{P}$ and $0.30 \mathrm{mmol}$ of $p$-toluenethiol for $3 \mathrm{~h}$.

在确定了最佳反应条件后(表 1, Entry 11)，对具有 不同取代基或取代模式的硝基类化合物 $\mathbf{1}$ 的反应普适性 进行了考察(表 2). 苯基的对位和间位带有供电子基团 和吸电子基团的硝基类化合物 $\mathbf{1}$ 都能发生还原反应，以 良好至优异的收率得到相应的目标化合物(表 2, 2b 21). 当苯基的邻位带有供电子基团或者吸电子基团时, 也能发生还原反应，但由于邻位取代基位阻较大，导致 
其相应的目标化合物收率都有所降低(表 2, 2m 2t). 随 后, 为了进一步研究该方法的普适性, 又尝试了硝基取 代的稠环芳烃菜和吡啶, 并分别以 $94 \%$ 和 $61 \%$ 的产率获 得了 1-氨基萘 $(2 \mathbf{u})$ 和 3 -氨基吡啶 $(\mathbf{2 v})$. 值得注意的是, 硝 基取代的三联苯也能发生还原反应，以 $82 \%$ 的收率得到 了目标产物 4-氨基对三联苯 $(2 \mathrm{w})$. 当使用 4,4-二硝基联 苯作为底物进行还原时, 以 $49 \%$ 的产率得到 4,4-二氨基 联苯 $(\mathbf{2 x})$. 遗憾的是, 当使用硝基环已烷参与反应时, 并没有生成目标化合物 (2y). 根据以上结果显示, 该方 法具有较为广泛的底物普适性.

表 2 硝基还原反应底物普适性研究

Table 2 Evaluation of substrate scope for the reduction of nitro the compounds

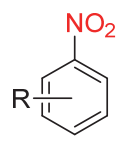

1

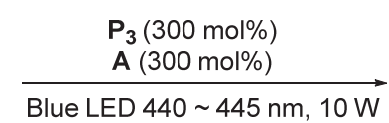

No solvent, r.t., $\mathrm{N}_{2}, 3 \mathrm{~h}$

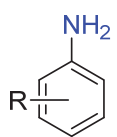

2

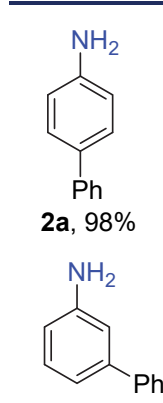

2g, $98 \%$

$\mathrm{NH}_{2}$<smiles>Nc1cccc(I)c1</smiles>

2I, $87 \%$<smiles>Nc1ccccc1Br</smiles>

2q, $35 \%$<smiles></smiles><smiles>Nc1ccc(Br)cc1</smiles>

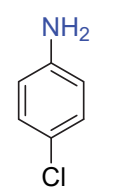

Cl

$\mathrm{NH}_{2}$

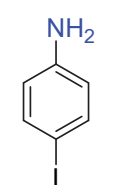

2f, $86 \%$<smiles>CC(C)(C)c1cccc(N)c1</smiles>

2h, $63 \%$<smiles>CCc1ccccc1N</smiles>

$2 \mathrm{~m}, 47 \%$<smiles>Nc1ccccc1Cl</smiles>

2r, $17 \%$<smiles>N#Cc1cccc(N)c1</smiles>

2i, $83 \%$<smiles>CCCc1ccccc1N</smiles>

2n, $30 \%$<smiles>Nc1ccccc1I</smiles>

2s, $38 \%$<smiles>Nc1cccc(Br)c1</smiles>

2j, $88 \%$<smiles>CC(C)(C)c1ccccc1N</smiles>

2o, $41 \%$<smiles>Nc1ccccc1C(F)(F)F</smiles>

2t, $45 \%$

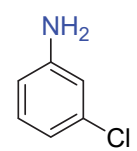

2k, $63 \%$ $\mathrm{NH}_{2}$<smiles>N#Cc1ccccc1N</smiles>

2 p, $40 \%$<smiles>Nc1cccc2ccccc12</smiles>

2u, $94 \%$

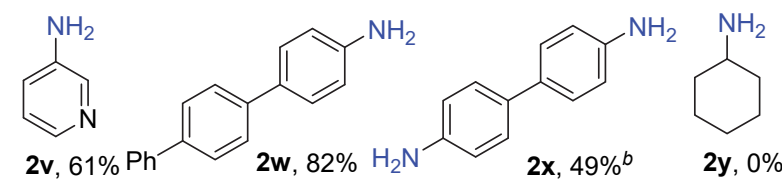

${ }^{a}$ General conditions: 1a $(0.10 \mathrm{mmol}), \mathbf{P}_{\mathbf{3}}(0.30 \mathrm{mmol}), \mathbf{A}(0.30 \mathrm{mmol})$, blue LED $440 \sim 445 \mathrm{~nm}$ and $10 \mathrm{~W}$ at r.t. for $3 \mathrm{~h} .{ }^{b} \mathbf{1 a}(0.10 \mathrm{mmol}), \mathbf{P}_{3}(0.60 \mathrm{mmol})$, A $(0.60 \mathrm{mmol})$, blue LED $440 \sim 445 \mathrm{~nm}$ and $10 \mathrm{~W}$ at r.t. for $3 \mathrm{~h}$

为了研究该反应的实用性, 以模型反应为例, 对反 应进行了 $1.0 \mathrm{mmol}$ 的放大实验 (Scheme 2a). 该还原反 应以 $88 \%$ 的收率得到目标产物 $\mathbf{2 a}$, 说明该反应具有较 好的实用性. 随后采用太阳光作为该还原反应的光源, 在不使用溶剂的情况下得到目标产物 2a, 收率为 $53 \%$ (Scheme 2b). 虽然收率有所降低，但提供了一条思路，

是否可以采用绿色清洁，经济易得的太阳光作为该还原 反应的光源，为合成胺类化合物提供一种绿色、经济、 可操作性强的新方法.

(a) $1.0 \mathrm{mmol}$ scales synthesis of $\mathbf{2 a}$

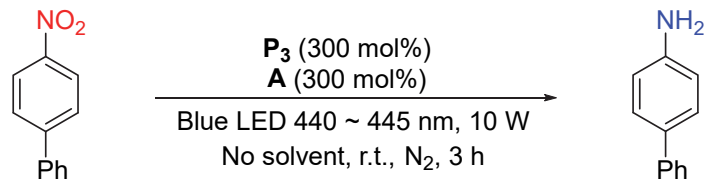

1a, $1.0 \mathrm{mmol}$

2a, $88 \%, 148 \mathrm{mg}$

(b) The reaction of sunlight as a light source<smiles>O=[N+]([O-])c1ccccc1</smiles>

$$
\begin{gathered}
\mathbf{P}_{3}(300 \mathrm{~mol} \%) \\
\text { A }(300 \mathrm{~mol} \%) \\
\text { Sun light, No solvent, r.t., } \mathrm{N}_{2}, 3 \mathrm{~h}
\end{gathered}
$$

$\mathrm{Ph}$

1a, $0.1 \mathrm{mmol}$

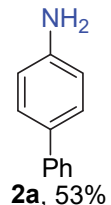

图式 $21.0 \mathrm{mmol}$ 规模的合成及太阳光作为光源的反应 Scheme 2 Synthesis at $1.0 \mathrm{mmol}$ scale and reaction using sunlight as light source

还原反应反应机理的推测如 Scheme 3 所示. 首先 三环已基膦进攻化合物 1a 中的硝基得到中间体 I, 还原 得到亚硝基 II, 然后三环己基膦攻击亚硝基中间体 II, 形成中间体 III. 这种富电子体系很容易消除三环己基 氧膦，形成氮烯中间体 $\mathbf{I V}^{[16]}$. 中间体 IV 非常不稳定， 可通过自由基过程从对甲苯硫酚中获取一个 $\mathrm{H}$ 原子，从 而形成富电子氮自由基 $\mathbf{V}$, 中间体 $\mathbf{V}$ 再次与一分子对甲 苯硫酚发生氢原子转移反应, 最终得到目标产物 2a. 值 得一提的是，被夺取了氢原子的两分子硫酚，可通过 A1 自由基中间体发生偶联反应生成对甲基苯二硫醚 A2. A2 分子在我们的反应体系中极不稳定, 非常容易 分解，但可以通过气相色谱一质谱联用手段对反应液进 行监测观察得到.

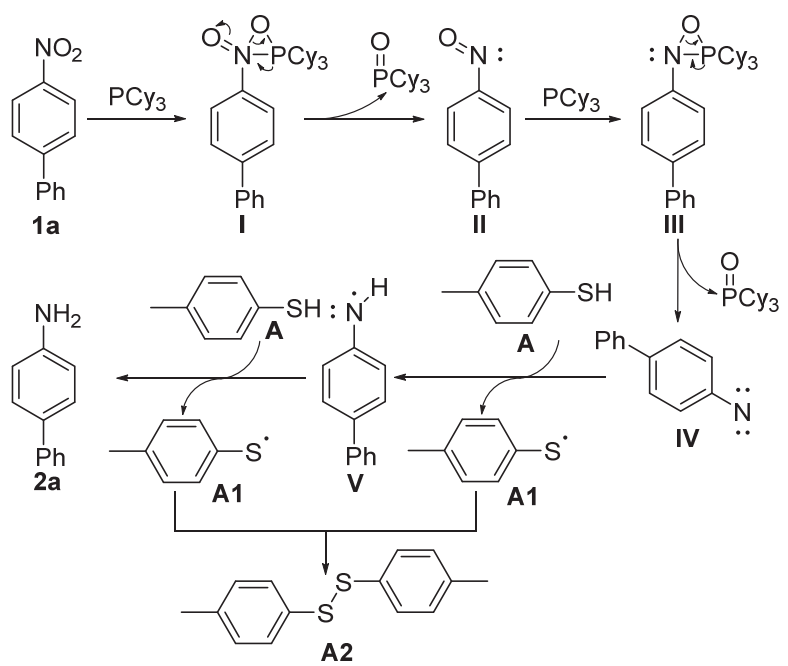

图式 3 推测的硝基还原反应机理

Scheme 3 Proposed reaction mechanism for the reduction of the nitro arene 


\section{2 结论}

综上所述, 开发了一种新型的无溶剂、无金属参与, 对甲苯硫酚/三环己基膦介导的硝基芳烃的光化学还原 合成胺类衍生物的方法. 该还原方法条件温和，底物适 用范围广，官能团耐受性优异且胺类衍生物可以获得良 好的产率. 还利用太阳光作为光源, 对该方法还原硝基 类化合物进行了初步研究, 这为今后开发合成胺类化合 物提供了一种绿色、经济、可操作性强的新策略, 并为 用于工业生产提供有益的参考价值.

\section{3 实验部分}

\section{1 仪器与试剂}

Bruker ASCEND (400 MHz) spectrometer 和 JEOL-ECX-500 (500 MHz)核磁共振仪, 以四甲基硅烷 (TMS)为内标, $\mathrm{CDCl}_{3}$ 为溶剂, $\mathrm{CDCl}_{3}$ 的化学位移值校正 为 $\delta 7.26\left({ }^{1} \mathrm{H} \mathrm{NMR}\right)$ 和 $77.16\left({ }^{13} \mathrm{C} \mathrm{NMR}\right)$. 所有的一阶分 裂模式都是根据多重峰的出现分配的, 不容易解释的分 裂模式被指定为多重峰 (m) 或宽峰 (br). WATTCAS 1020SL 光化学反应仪; Thermo Fisher Q Exactive mass spectrometer 高分辨质谱仪(赛默飞世尔科技有限公司); WFH-203B 型三用紫外分析仪(上海精科实业有限公司). 用 $0.2 \mathrm{~mm}$ 的硅胶板(TLC)来监测反应, 在紫外灯下进行 观测.

所有的试剂和底物均是从市场直接购买的, 试剂的 厂家有安耐吉化学、毕得医药、乐研、百灵威等.

\section{2 实验方法}

在 $4.0 \mathrm{~mL}$ 的反应瓶中依次加入 $1(0.10 \mathrm{mmol})$ 、三 环己基膦 $(0.30 \mathrm{mmol})$ 、对甲基苯硫酚 $(0.30 \mathrm{mmol})$, 混合 均匀, 将光化学反应仪调节成 $10 \mathrm{~W}, 3 \mathrm{~h}$, 开启光源 $(440 \sim 445 \mathrm{~nm})$. 反应结束后, 用 $0.5 \mathrm{~mL}$ 的二氯甲烷 (DCM) 溶解后, 用 $100 \sim 200$ 目的硅胶, 经柱层析 $[V($ 石 油醚 $): V($ 乙酸乙酯 $)=50 ： 1 \sim 10 ： 1]$ 纯化得到目标化 合物 $\mathbf{2 a} \sim \mathbf{2 x}$.

[1,1'-联苯]-4-胺 $(2 \mathbf{a})^{[17]}$ : 白色固体, $16.6 \mathrm{mg}$, 收率 98\%. m.p. 51 53 ${ }^{\circ} \mathrm{C}$; ${ }^{1} \mathrm{H}$ NMR (400 MHz, $\left.\mathrm{CDCl}_{3}\right) \delta$ : $7.57 \sim 7.50(\mathrm{~m}, 2 \mathrm{H}), 7.45 \sim 7.35(\mathrm{~m}, 4 \mathrm{H}), 7.25(\mathrm{~s}, 1 \mathrm{H})$, $6.79 \sim 6.72(\mathrm{~m}, 2 \mathrm{H}), 3.72\left(\mathrm{~s}, 2 \mathrm{H}, \mathrm{NH}_{2}\right) ;{ }^{13} \mathrm{C} \mathrm{NMR}(101$ $\left.\mathrm{MHz}, \mathrm{CDCl}_{3}\right) \delta: 145.9,141.2,131.6,128.7,128.0,126.4$, 126.3, 115.4. HRMS calcd for $\mathrm{C}_{12} \mathrm{H}_{12} \mathrm{~N}[\mathrm{M}+\mathrm{H}]^{+}$ 170.0964, found 170.0967 .

4-异丙基苯胺 $(\mathbf{2 b})^{[5 \mathrm{~h}]}$ : 淡黄色液体, $8.0 \mathrm{mg}$, 收率 $59 \%$. ${ }^{1} \mathrm{H}$ NMR (400 MHz, $\left.\mathrm{CDCl}_{3}\right) \delta: 7.1 \sim 7.0(\mathrm{~m}, 2 \mathrm{H})$, $6.7 \sim 6.6(\mathrm{~m}, 2 \mathrm{H}), 3.1\left(\mathrm{~s}, 2 \mathrm{H}, \mathrm{NH}_{2}\right), 2.75 \sim 2.86(\mathrm{~m}, 1 \mathrm{H}$, $\left.\mathrm{CH}\left(\mathrm{CH}_{3}\right)_{2}\right), 1.2\left(\mathrm{~d}, J=8.0 \mathrm{~Hz}, 6 \mathrm{H}, \mathrm{CH}\left(\mathrm{CH}_{3}\right)_{2}\right) ;{ }^{13} \mathrm{C} \mathrm{NMR}$ $\left(101 \mathrm{MHz}, \mathrm{CDCl}_{3}\right) \delta: 144.1,139.2,127.2,115.2,33.2$, 24.2. HRMS calcd for $\mathrm{C}_{9} \mathrm{H}_{14} \mathrm{~N}[\mathrm{M}+\mathrm{H}]^{+}$136.1121, found 136.1123 .

4-氨基苯甲腈 $(2 \mathrm{c})^{[5 \mathrm{~h}]}$ : 白色固体, $7.4 \mathrm{mg}$, 收率 $63 \%$. m.p. 83 85 ${ }^{\circ} \mathrm{C} ;{ }^{1} \mathrm{H}$ NMR $\left(400 \mathrm{MHz}, \mathrm{CDCl}_{3}\right) \delta: 7.46 \sim$ $7.33(\mathrm{~m}, 2 \mathrm{H}), 6.72 \sim 6.57(\mathrm{~m}, 2 \mathrm{H}), 4.22\left(\mathrm{~s}, 2 \mathrm{H}, \mathrm{NH}_{2}\right) ;{ }^{13} \mathrm{C}$ NMR (101 MHz, $\left.\mathrm{CDCl}_{3}\right) \delta: 150.6,133.8,120.3,114.5$, 100.0. HRMS calcd for $\mathrm{C}_{7} \mathrm{H}_{7} \mathrm{~N}_{2}[\mathrm{M}+\mathrm{H}]^{+} 119.0604$, found 119.0608 .

4-溴苯胺 $(2 d)^{[17]}$ : 白色固体， $16.9 \mathrm{mg}$, 收率 $98 \%$. m.p. $59 \sim 61{ }^{\circ} \mathrm{C} ;{ }^{1} \mathrm{H}$ NMR $\left(400 \mathrm{MHz}, \mathrm{CDCl}_{3}\right) \delta: 7.25 \sim$ $7.18(\mathrm{~m}, 2 \mathrm{H}), 6.66 \sim 6.46(\mathrm{~m}, 2 \mathrm{H}), 3.61\left(\mathrm{~s}, 2 \mathrm{H}, \mathrm{NH}_{2}\right) ;{ }^{13} \mathrm{C}$ NMR (101 MHz, $\left.\mathrm{CDCl}_{3}\right) \delta: 145.4,132.0,116.8,110.2$. HRMS calcd for $\mathrm{C}_{6} \mathrm{H}_{7} \mathrm{BrN}[\mathrm{M}+\mathrm{H}]^{+}$171.9756, found 171.9759 .

4-氯苯胺 $(2 \mathrm{e})^{[17]}$ : 白色固体， $10.1 \mathrm{mg}$, 收率 $79 \%$. m.p. $66 \sim 69{ }^{\circ} \mathrm{C} ;{ }^{1} \mathrm{H}$ NMR $\left(400 \mathrm{MHz}, \mathrm{CDCl}_{3}\right) \delta: 7.17 \sim$ $7.03(\mathrm{~m}, 2 \mathrm{H}), 6.66 \sim 6.52(\mathrm{~m}, 2 \mathrm{H}), 3.44\left(\mathrm{~s}, 2 \mathrm{H}, \mathrm{NH}_{2}\right) ;{ }^{13} \mathrm{C}$ NMR (101 MHz, $\left.\mathrm{CDCl}_{3}\right) \delta: 145.0,129.1,123.2,116.3$. HRMS calcd for $\mathrm{C}_{6} \mathrm{H}_{7} \mathrm{ClN}[\mathrm{M}+\mathrm{H}]^{+}$128.0262, found 128.0266 .

4-碘苯胺 $(2 \mathrm{f})^{[17]}$ : 白色固体， $18.8 \mathrm{mg}$, 收率 $86 \%$. m.p. $60 \sim 61{ }^{\circ} \mathrm{C} ;{ }^{1} \mathrm{H}$ NMR (400 $\left.\mathrm{MHz}, \mathrm{CDCl}_{3}\right) \delta: 7.48 \sim$ $7.35(\mathrm{~m}, 2 \mathrm{H}), 6.53 \sim 6.39(\mathrm{~m}, 2 \mathrm{H}), 3.40\left(\mathrm{~s}, 2 \mathrm{H}, \mathrm{NH}_{2}\right) ;{ }^{13} \mathrm{C}$ NMR (101 MHz, $\left.\mathrm{CDCl}_{3}\right) \delta: 146.1,137.9,117.3,79.4$. HRMS calcd for $\mathrm{C}_{6} \mathrm{H}_{7} \mathrm{IN}[\mathrm{M}+\mathrm{H}]^{+}$219.9618, found 219.9621 .

[1,1'-联苯]-3-胺 $(2 \mathrm{~g})^{[18]}$ : 淡黄色液体, $16.6 \mathrm{mg}$, 收率 98\%. ${ }^{1} \mathrm{H}$ NMR (400 MHz, $\left.\mathrm{CDCl}_{3}\right) \delta: 7.59 \sim 7.52(\mathrm{~m}, 2 \mathrm{H})$, $7.47 \sim 7.36(\mathrm{~m}, 2 \mathrm{H}), 7.36 \sim 7.28(\mathrm{~m}, 1 \mathrm{H}), 7.26 \sim 7.16(\mathrm{~m}$, $1 \mathrm{H}), 7.03 \sim 6.95(\mathrm{~m}, 1 \mathrm{H}), 6.89(\mathrm{t}, J=2.0 \mathrm{~Hz}, 1 \mathrm{H}), 6.66$ (ddd, $J=7.9,2.4,1.0 \mathrm{~Hz}, 1 \mathrm{H}), 3.44\left(\mathrm{~s}, 2 \mathrm{H}, \mathrm{NH}_{2}\right) ;{ }^{13} \mathrm{C}$ NMR $\left(101 \mathrm{MHz}, \mathrm{CDCl}_{3}\right) \delta: 146.8,142.5,141.4,129.7$, $128.7,127.3,127.2,117.7,114.2,114.0$. HRMS calcd for $\mathrm{C}_{12} \mathrm{H}_{12} \mathrm{~N}[\mathrm{M}+\mathrm{H}]^{+}$170.0964, found 170.0960.

3-叔丁基苯胺(2h)：无色液体，9.4 mg，收率 63\%. ${ }^{1} \mathrm{H}$ NMR (400 MHz, $\left.\mathrm{CDCl}_{3}\right) \delta: 7.09(\mathrm{t}, J=7.8 \mathrm{~Hz}, 1 \mathrm{H})$, 6.80 (ddd, $J=7.9,1.9,1.0 \mathrm{~Hz}, 1 \mathrm{H}), 6.72(\mathrm{t}, J=2.1 \mathrm{~Hz}$, 1H), 6.52 (ddd, $J=7.8,2.3,1.0 \mathrm{~Hz}, 1 \mathrm{H}), 3.35(\mathrm{~s}, 2 \mathrm{H}$, $\left.\mathrm{NH}_{2}\right), 1.29$ (s, 9H, C(CH3) $\left.)_{3}\right) ;{ }^{13} \mathrm{C} \mathrm{NMR} \mathrm{(101} \mathrm{MHz,} \mathrm{CDCl}_{3}$ ) $\delta: 152.5,146.1,129.0,115.9,112.5,112.4,34.6,31.3$. HRMS calcd for $\mathrm{C}_{10} \mathrm{H}_{16} \mathrm{~N}[\mathrm{M}+\mathrm{H}]^{+}$150.1277, found 150.1279 .

3-氨基苯甲腈 $(\mathbf{2 i})^{[15]}$ : 淡黄色液体, $9.8 \mathrm{mg}$, 收率 $83 \%$. ${ }^{1} \mathrm{H}$ NMR $\left(400 \mathrm{MHz}, \mathrm{CDCl}_{3}\right) \delta: 7.22(\mathrm{t}, J=7.9 \mathrm{~Hz}$, 
1H), $7.01(\mathrm{~d}, J=7.6 \mathrm{~Hz}, 1 \mathrm{H}), 6.96 \sim 6.76(\mathrm{~m}, 2 \mathrm{H}), 4.40 \sim$ $3.32\left(\mathrm{~m}, 2 \mathrm{H}, \mathrm{NH}_{2}\right) ;{ }^{13} \mathrm{C} \mathrm{NMR}\left(101 \mathrm{MHz}, \mathrm{CDCl}_{3}\right) \delta: 147.0$, 130.1, 122.0, 119.2, 117.4, 112.9. HRMS calcd for $\mathrm{C}_{7} \mathrm{H}_{7} \mathrm{~N}_{2}$ $[\mathrm{M}+\mathrm{H}]^{+} 119.0604$, found 119.0607 .

3-溴苯胺 $(\mathbf{2 j})^{[17]}$ : 淡黄色液体, $15.1 \mathrm{mg}$, 收率 $88 \%$. ${ }^{1} \mathrm{H}$ NMR $\left(400 \mathrm{MHz}, \mathrm{CDCl}_{3}\right) \delta: 6.99$ (t, $\left.J=7.9 \mathrm{~Hz}, 1 \mathrm{H}\right)$, $6.90 \sim 6.79(\mathrm{~m}, 2 \mathrm{H}), 6.58(\mathrm{ddd}, J=8.0,2.2,1.0 \mathrm{~Hz}, 1 \mathrm{H})$, $3.61\left(\mathrm{~s}, 2 \mathrm{H}, \mathrm{NH}_{2}\right) ;{ }^{13} \mathrm{C}$ NMR $\left(101 \mathrm{MHz}, \mathrm{CDCl}_{3}\right) \delta: 147.8$, 130.6, 123.1, 121.4, 117.8, 113.7. HRMS calcd for $\mathrm{C}_{6} \mathrm{H}_{7} \mathrm{BrN}[\mathrm{M}+\mathrm{H}]^{+} \quad$ 171.9756, found 171.9760 .

3 -氯苯胺 $(\mathbf{2 k})^{[19]}$ : 淡黄色液体, $8.0 \mathrm{mg}$, 收率 $63 \%$. ${ }^{1} \mathrm{H}$ NMR $\left(400 \mathrm{MHz}, \mathrm{CDCl}_{3}\right) \delta: 7.00(\mathrm{t}, J=8.0 \mathrm{~Hz}, 1 \mathrm{H})$, $6.72 \sim 6.65(\mathrm{~m}, 1 \mathrm{H}), 6.58(\mathrm{t}, J=2.2 \mathrm{~Hz}, 1 \mathrm{H}), 6.49 \sim 6.43$ (m, $1 \mathrm{H}), 3.60$ (s, $\left.2 \mathrm{H}, \mathrm{NH}_{2}\right) ;{ }^{13} \mathrm{C}$ NMR $\left(101 \mathrm{MHz}, \mathrm{CDCl}_{3}\right.$ ) $\delta: 147.9,134.8,130.5,118.4,115.0,113.4$. HRMS calcd for $\mathrm{C}_{6} \mathrm{H}_{7} \mathrm{ClN}[\mathrm{M}+\mathrm{H}]^{+}$128.0262, found 128.0260 .

3-碘苯胺 $(2 \mathrm{I})^{[15]}$ : 淡黄色液体, $19.1 \mathrm{mg}$, 收率 $87 \%$. ${ }^{1} \mathrm{H}$ NMR (400 MHz, $\left.\mathrm{CDCl}_{3}\right) \delta: 7.11 \sim 7.00(\mathrm{~m}, 2 \mathrm{H}), 6.85$ (t, $J=7.9 \mathrm{~Hz}, 1 \mathrm{H}$ ), 6.61 (ddd, $J=8.1,2.3,1.0 \mathrm{~Hz}, 1 \mathrm{H}$ ), 3.45 (s, $\left.2 \mathrm{H}, \mathrm{NH}_{2}\right) ;{ }^{13} \mathrm{C} \mathrm{NMR}\left(101 \mathrm{MHz}, \mathrm{CDCl}_{3}\right) \delta: 147.8$, $130.8,127.5,123.8,114.3,95.0$. HRMS calcd for $\mathrm{C}_{6} \mathrm{H}_{7} \mathrm{IN}$ $[\mathrm{M}+\mathrm{H}]^{+}$219.9618, found 219.9621.

2-乙基苯胺 $(\mathbf{2 m})^{[20]}$ : 无色液体, $5.7 \mathrm{mg}$, 收率 $47 \%$. ${ }^{1} \mathrm{H}$ NMR $\left(400 \mathrm{MHz}, \mathrm{CDCl}_{3}\right) \delta: 7.10 \sim 6.99(\mathrm{~m}, 2 \mathrm{H}), 6.74$ (td, $J=7.4,1.3 \mathrm{~Hz}, 1 \mathrm{H}), 6.65$ (dd, $J=7.9,1.3 \mathrm{~Hz}, 1 \mathrm{H})$, 3.53 (s, $\left.2 \mathrm{H}, \mathrm{NH}_{2}\right), 2.50$ (q, $\left.J=7.5 \mathrm{~Hz}, 2 \mathrm{H}, \mathrm{CH}_{2} \mathrm{CH}_{3}\right), 1.24$ (t, $\left.J=7.6 \mathrm{~Hz}, 3 \mathrm{H}, \mathrm{CH}_{2} \mathrm{CH}_{3}\right) ;{ }^{13} \mathrm{C} \mathrm{NMR}\left(101 \mathrm{MHz}, \mathrm{CDCl}_{3}\right)$ $\delta$ : 144.1, 128.4, 128.1, 126.9, 118.9, 115.4, 24.1, 13.1. HRMS calcd for $\mathrm{C}_{8} \mathrm{H}_{12} \mathrm{~N}[\mathrm{M}+\mathrm{H}]^{+}$122.0964, found 122.0958.

2-异丙基苯胺 $(2 \mathbf{n})^{[21]}$ : 淡黄色液体, $4.1 \mathrm{mg}$, 收率 30\%. ${ }^{1} \mathrm{H}$ NMR $\left(400 \mathrm{MHz}, \mathrm{CDCl}_{3}\right) \delta: 7.13(\mathrm{~d}, J=7.7 \mathrm{~Hz}$, $1 \mathrm{H}), 7.03 \sim 6.98(\mathrm{~m}, 1 \mathrm{H}), 6.77$ (td, $J=7.5,1.5 \mathrm{~Hz}, 1 \mathrm{H})$, $6.64(\mathrm{dd}, J=7.9,1.4 \mathrm{~Hz}, 1 \mathrm{H}), 3.60\left(\mathrm{~s}, 2 \mathrm{H}, \mathrm{NH}_{2}\right), 2.93 \sim$ $2.83\left(\mathrm{~m}, 1 \mathrm{H}, \mathrm{CH}\left(\mathrm{CH}_{3}\right)_{2}\right), 1.25(\mathrm{dd}, J=6.9,1.3 \mathrm{~Hz}, 6 \mathrm{H}$, $\left.\mathrm{CH}\left(\mathrm{CH}_{3}\right)_{2}\right) ;{ }^{13} \mathrm{C} \mathrm{NMR}\left(101 \mathrm{MHz}, \mathrm{CDCl}_{3}\right) \delta: 143.4,132.7$, 126.6, 125.5, 119.1, 115.9, 27.7, 22.4. HRMS calcd for $\mathrm{C}_{9} \mathrm{H}_{14} \mathrm{~N}[\mathrm{M}+\mathrm{H}]^{+}$136.1121, found 136.1121 .

2-叔丁基苯胺(2o): 淡黄色液体, $6.1 \mathrm{mg}$, 收率 41\%. ${ }^{1} \mathrm{H}$ NMR $\left(400 \mathrm{MHz}, \mathrm{CDCl}_{3}\right) \delta: 7.23(\mathrm{dd}, J=7.9,1.6 \mathrm{~Hz}$, $1 \mathrm{H}), 7.02(\mathrm{td}, J=7.5,1.5 \mathrm{~Hz}, 1 \mathrm{H}), 6.73(\mathrm{td}, J=7.6,1.4 \mathrm{~Hz}$, $1 \mathrm{H}), 6.62(\mathrm{dd}, J=7.8,1.4 \mathrm{~Hz}, 1 \mathrm{H}), 3.73\left(\mathrm{~s}, 2 \mathrm{H}, \mathrm{NH}_{2}\right), 1.41$ (s, 9H, $\left.\mathrm{C}\left(\mathrm{CH}_{3}\right)_{3}\right) ;{ }^{13} \mathrm{C}$ NMR (101 MHz, $\left.\mathrm{CDCl}_{3}\right) \delta: 144.7$, 133.8, 127.1, 126.6, 118.7, 117.9, 34.3, 29.7. HRMS calcd for $\mathrm{C}_{10} \mathrm{H}_{16} \mathrm{~N}[\mathrm{M}+\mathrm{H}]^{+} \quad 150.1277$, found 150.1278 . 2-氨基苯甲腈(2p) ${ }^{[15]}$ : 白色固体, $4.7 \mathrm{mg}$, 收率 $40 \%$. m.p. $46 \sim 47{ }^{\circ} \mathrm{C} ;{ }^{1} \mathrm{H}$ NMR $\left(400 \mathrm{MHz}, \mathrm{CDCl}_{3}\right) \delta: 7.38(\mathrm{dd}$, $J=8.0,1.6 \mathrm{~Hz}, 1 \mathrm{H}), 7.32(\mathrm{ddd}, J=8.9,7.3,1.6 \mathrm{~Hz}, 1 \mathrm{H})$, 6.79 6.69 (m, 2H), $4.41\left(\mathrm{~s}, 2 \mathrm{H}, \mathrm{NH}_{2}\right) ;{ }^{13} \mathrm{C}$ NMR $(101$ $\left.\mathrm{MHz}, \mathrm{CDCl}_{3}\right) \delta: 149.6,134.0,132.4,118.0,117.6,115.2$, 96.1. HRMS calcd for $\mathrm{C}_{7} \mathrm{H}_{7} \mathrm{~N}_{2}[\mathrm{M}+\mathrm{H}]^{+}$119.0604, found 119.0607 .

2-溴苯胺 $(2 q)^{[22]}$ : 淡黄色液体, $6.0 \mathrm{mg}$, 收率 $35 \%$. ${ }^{1} \mathrm{H}$ NMR $\left(400 \mathrm{MHz}, \mathrm{CDCl}_{3}\right) \delta: 7.39(\mathrm{dt}, J=8.0,1.6 \mathrm{~Hz}$, $1 \mathrm{H}), 7.08(\mathrm{td}, J=7.5,1.4 \mathrm{~Hz}, 1 \mathrm{H}), 6.72(\mathrm{dd}, J=8.0,1.6$ $\mathrm{Hz}, 1 \mathrm{H}), 6.59(\mathrm{td}, J=7.5,1.5 \mathrm{~Hz}, 1 \mathrm{H}), 4.04\left(\mathrm{~s}, 2 \mathrm{H}, \mathrm{NH}_{2}\right)$; ${ }^{13} \mathrm{C}$ NMR (101 MHz, $\left.\mathrm{CDCl}_{3}\right) \delta: 144.2,132.6,128.4,119.5$, 115.9, 109.4. HRMS calcd for $\mathrm{C}_{6} \mathrm{H}_{7} \mathrm{BrN}[\mathrm{M}+\mathrm{H}]^{+}$ 171.9756, found 171.9757 .

2-氯苯胺 $(2 \mathbf{r})^{[19]}$ : 淡黄色液体, $2.2 \mathrm{mg}$, 收率 $17 \%$. ${ }^{1} \mathrm{H}$ NMR $\left(400 \mathrm{MHz}, \mathrm{CDCl}_{3}\right) \delta: 7.23(\mathrm{dd}, J=7.9,1.4 \mathrm{~Hz}$, $1 \mathrm{H}), 7.05(\mathrm{td}, J=7.7,1.5 \mathrm{~Hz}, 1 \mathrm{H}), 6.74(\mathrm{dd}, J=8.0,1.6$ $\mathrm{Hz}, 1 \mathrm{H}), 6.67$ (td, $J=7.7,1.5 \mathrm{~Hz}, 1 \mathrm{H}), 3.99$ (s, $2 \mathrm{H}, \mathrm{NH}_{2}$ ); ${ }^{13} \mathrm{C}$ NMR (101 MHz, $\left.\mathrm{CDCl}_{3}\right) \delta: 143.0,129.5,127.7,119.3$, 119.1, 115.9. HRMS calcd for $\mathrm{C}_{6} \mathrm{H}_{7} \mathrm{ClN}[\mathrm{M}+\mathrm{H}]^{+}$ 128.0262, found 128.0257 .

2-碘苯胺 $(2 \mathrm{~s})^{[15]}$ : 淡黄色液体, $8.3 \mathrm{mg}$, 收率 $38 \% .{ }^{1} \mathrm{H}$ NMR $\left(400 \mathrm{MHz}, \mathrm{CDCl}_{3}\right) \delta: 7.63(\mathrm{dd}, J=7.9,1.4 \mathrm{~Hz}, 1 \mathrm{H})$, 7.13 (ddd, $J=8.0,7.3,1.5 \mathrm{~Hz}, 1 \mathrm{H}$ ), 6.75 (dd, $J=8.0,1.5$ $\mathrm{Hz}, 1 \mathrm{H}$ ), 6.47 (ddd, $J=7.9,7.2,1.5 \mathrm{~Hz}, 1 \mathrm{H}), 4.06$ (s, 2H, $\mathrm{NH}_{2}$ ); ${ }^{13} \mathrm{C}$ NMR (101 MHz, $\left.\mathrm{CDCl}_{3}\right) \delta: 146.8,139.0,129.4$, 120.0, 114.8, 84.2. HRMS calcd for $\mathrm{C}_{6} \mathrm{H}_{7} \mathrm{IN}[\mathrm{M}+\mathrm{H}]^{+}$ 219.9618 , found 219.9617 .

2-三氟甲基苯胺(2t): 无色液体, $7.2 \mathrm{mg}$, 收率 45\%. ${ }^{1} \mathrm{H}$ NMR $\left(400 \mathrm{MHz}, \mathrm{CDCl}_{3}\right) \delta: 7.4(\mathrm{~d}, J=8.0 \mathrm{~Hz}, 1 \mathrm{H}), 7.3$ (ddd, $J=8.8,7.6,1.5 \mathrm{~Hz}, 1 \mathrm{H}), 6.8 \sim 6.7(\mathrm{~m}, 2 \mathrm{H}), 4.1(\mathrm{~s}$, $2 \mathrm{H}) ;{ }^{13} \mathrm{C}$ NMR (101 MHz, $\left.\mathrm{CDCl}_{3}\right) \delta: 144.5,132.9,126.5$ (q, $J=5.3 \mathrm{~Hz}), 125.1$ (q, $J=272.2 \mathrm{~Hz}), 117.6,117.1113 .8$ $(\mathrm{q}, J=30 \mathrm{~Hz}) ;{ }^{19} \mathrm{~F}$ NMR $\left(376 \mathrm{MHz}, \mathrm{CDCl}_{3}\right) \delta:-62.8$. HRMS calcd for $\mathrm{C}_{7} \mathrm{H}_{5} \mathrm{~F}_{3} \mathrm{~N}[\mathrm{M}-\mathrm{H}]^{-} 160.0380$, found 160.0380 .

1-氨基萗 $(\mathbf{2 u})^{[17]}$ : 白色固体, $13.5 \mathrm{mg}$, 收率 $94 \%$. m.p. $48 \sim 49{ }^{\circ} \mathrm{C}$; ${ }^{1} \mathrm{H}$ NMR $\left(400 \mathrm{MHz}, \mathrm{CDCl}_{3}\right) \delta: 7.81 \sim$ $7.70(\mathrm{~m}, 2 \mathrm{H}), 7.47 \sim 7.36(\mathrm{~m}, 2 \mathrm{H}), 7.32 \sim 7.21(\mathrm{~m}, 2 \mathrm{H})$, $6.70(\mathrm{dd}, J=7.0,1.5 \mathrm{~Hz}, 1 \mathrm{H}), 3.95\left(\mathrm{~s}, 2 \mathrm{H}, \mathrm{NH}_{2}\right) ;{ }^{13} \mathrm{C} \mathrm{NMR}$ $\left(101 \mathrm{MHz}, \mathrm{CDCl}_{3}\right) \delta: 142.2,134.5,128.7,126.5,126.0$, 125.0, 123.8, 120.9, 119.0, 109.8. HRMS calcd for $\mathrm{C}_{10} \mathrm{H}_{10} \mathrm{~N}[\mathrm{M}+\mathrm{H}]^{+}$144.0808, found 144.0808.

3-氨基吡啶 $(2 \mathrm{v})^{[17]}$ : 白色固体, $5.7 \mathrm{mg}$, 收率 $61 \%$. m.p. 59 60 ${ }^{\circ} \mathrm{C} ;{ }^{1} \mathrm{H}$ NMR (400 MHz, $\left.\mathrm{CDCl}_{3}\right) \delta: 8.09$ (d, 
$J=2.7 \mathrm{~Hz}, 1 \mathrm{H}), 8.01(\mathrm{dd}, J=4.7,1.6 \mathrm{~Hz}, 1 \mathrm{H}), 7.06(\mathrm{dd}$, $J=8.1,4.7 \mathrm{~Hz}, 1 \mathrm{H}), 6.96(\mathrm{ddd}, J=8.2,3.0,1.5 \mathrm{~Hz}, 1 \mathrm{H})$, $3.71\left(\mathrm{~s}, 2 \mathrm{H}, \mathrm{NH}_{2}\right) ;{ }^{13} \mathrm{C} \mathrm{NMR}\left(101 \mathrm{MHz}, \mathrm{CDCl}_{3}\right) \delta: 142.7$, 139.9, 137.5, 123.8, 121.5. HRMS calcd for $\mathrm{C}_{5} \mathrm{H}_{7} \mathrm{~N}_{2}[\mathrm{M}+$ $\mathrm{H}]^{+}$95.0604, found 95.0609.

4-氨基对三联苯 $(\mathbf{2 w})$ : 白色固体, $20.1 \mathrm{mg}$, 收率 $82 \%$. m.p. $200 \sim 201{ }^{\circ} \mathrm{C}$; ${ }^{1} \mathrm{H}$ NMR (400 MHz, $\left.\mathrm{CDCl}_{3}\right) \delta$ : $7.63(\mathrm{dd}, J=7.5,2.0 \mathrm{~Hz}, 6 \mathrm{H}), 7.50 \sim 7.40(\mathrm{~m}, 4 \mathrm{H}), 7.34(\mathrm{t}$, $J=7.3 \mathrm{~Hz}, 1 \mathrm{H}), 6.81 \sim 6.73(\mathrm{~m}, 2 \mathrm{H}), 3.74\left(\mathrm{~s}, 2 \mathrm{H}, \mathrm{NH}_{2}\right)$; ${ }^{13} \mathrm{C}$ NMR (101 MHz, $\left.\mathrm{CDCl}_{3}\right) \delta: 146.0,140.9,140.1,139.1$, $131.0,128.8,128.0,127.4,127.2,127.0,126.7,115.5$. HRMS calcd for $\mathrm{C}_{18} \mathrm{H}_{16} \mathrm{~N}[\mathrm{M}+\mathrm{H}]^{+}$246.1277, found 246.1275 .

4,4'-二氨基联苯 $(\mathbf{2 x})$ : 白色固体, $9.1 \mathrm{mg}$, 收率 49\%. m.p. $118 \sim 120{ }^{\circ} \mathrm{C} ;{ }^{1} \mathrm{H}$ NMR (400 MHz, $\left.\mathrm{CDC}_{13}\right) \delta: 7.4 \sim$ $7.3(\mathrm{~m}, 4 \mathrm{H}), 6.8 \sim 6.7(\mathrm{~m}, 4 \mathrm{H}), 3.6\left(\mathrm{~s}, 4 \mathrm{H}, \mathrm{NH}_{2}\right) ;{ }^{13} \mathrm{C} \mathrm{NMR}$ $\left(101 \mathrm{MHz}, \mathrm{CDCl}_{3}\right) \delta: 145.0,131.9,127.3,115.5$. HRMS calcd for $\mathrm{C}_{12} \mathrm{H}_{13} \mathrm{~N}_{2}[\mathrm{M}+\mathrm{H}]^{+} \quad 185.1073$, found 185.1075.

辅助材料(Supporting Information) 合成化合物的 ${ }^{1} \mathrm{H}$ $\mathrm{NMR},{ }^{13} \mathrm{C}$ NMR, ${ }^{19} \mathrm{~F}$ NMR 谱图. 这些材料可以免费从本 刊网站(http:// sioc-journal.cn/)上下载.

\section{References}

[1] (a) Zhu, S.; Xu, S.; Jing, W.; Zhao, Z.; Jiang, J. J. Agric. Food Chem. 2016, 64, 9702 .

(b) Liu, X. H.; Wen, Y. H.; Cheng, L.; Xu, T. M.; Wu, N. J. J. Agric. Food Chem. 2021, 69, 6968.

[2] (a) Ye, L. J.; Toh, H. H.; Yang, Y.; Adams, J. P.; Snajdrova, R.; Li, Z. ACS Catal. 2015, 5,1119 .

(b) Werner, L.; Machara, A.; Hudlicky, T. Adv. Synth. Catal. 2010, $352,195$.

(c) Das Sarma, K.; Zhang, J.; Huang, Y.; Davidson, J. G. Eur. J. Org. Chem. 2006, 2006, 3730.

[3] (a) Lin, C.-F.; Chien, C.-W.; Ojima, I. Org. Chem. Front. 2014, 1, 1062 .

(b) Afanasyev, O. I.; Kuchuk, E.; Usanov, D. L.; Chusov, D. Chem. Rev. 2019, 119, 11857

[4] (a) Strotman, N. A.; Baxter, C. A.; Brands, K. M.; Cleator, E.; Krska, S. W.; Reamer, R. A.; Wallace, D. J.; Wright, T. J. J. Am. Chem. Soc. 2011, 133, 8362.

(b) Magnus, P.; Sane, N.; Fauber, B. P.; Lynch, V. J. Am. Chem. Soc. 2009, 131, 16045.

(c) Cox, C. D.; Breslin, M. J.; Whitman, D. B.; Schreier, J. D.; McGaughey, G. B.; Bogusky, M. J.; Roecker, A. J.; Mercer, S. P.; Bednar, R. A.; Lemaire, W.; Bruno, J. G.; Reiss, D. R.; Harrell, C. M.; Murphy, K. L.; Garson, S. L.; Doran, S. M.; Prueksaritanont, T.; Anderson, W. B.; Tang, C.; Roller, S.; Cabalu, T. D.; Cui, D.; Hartman, G. D.; Young, S. D.; Koblan, K. S.; Winrow, C. J.; Renger, J. J.; Coleman, P. J. J. Med. Chem. 2010, 53, 5320.

(d) Baxter, C. A.; Cleator, E.; Brands, K. M. J.; Edwards, J. S.; Reamer, R. A.; Sheen, F. J.; Stewart, G. W.; Strotman, N. A.; Wallace, D. J. Org. Process Res. Dev. 2011, 15, 367.

[5] (a) Xu, D.-Q.; Hu, Z.-Y.; Li, W.-W.; Luo, S.-P.; Xu, Z.-Y. J. Mol. Catal. A: Chem. 2005, 235, 137.

(b) Wu, G.; Huang, M.; Richards, M.; Poirier, M.; Wen, X.; Draper,
R. W. Synthesis 2003, 1657.

(c) Xu, S.; Tang, J.; Zhou, Q.; Du, J.; Li, H. ACS Sustainable Chem. Eng. 2019, 7, 16190.

(d) Xiao, Q.; Sarina, S.; Waclawik, E. R.; Jia, J.; Chang, J.; Riches, J. D.; Wu, H.; Zheng, Z.; Zhu, H. ACS Catal. 2016, 6, 1744.

(e) Tsutsumi, K.; Uchikawa, F.; Sakai, K.; Tabata, K. ACS Catal. 2016, 6, 4394.

(f) Park, Y.; Kim, Y.; Chang, S. Chem. Rev. 2017, 117, 9247.

(g) Pachisia, S.; Kishan, R.; Yadav, S.; Gupta, R. Inorg. Chem. 2021, 60, 2009.

(h) Liu, Y.; Miao, W.; Tang, W.; Xue, D.; Xiao, J.; Wang, C.; Li, C. Chem. Asian J. 2021, 16, 1725.

(i) Gutiérrez-Tarriño, S.; Rojas-Buzo, S.; Lopes, C. W.; Agostini, G.; Calvino, J. J.; Corma, A.; Oña-Burgos, P. Green Chem. 2021, 23, 4490 .

(j) Cheung, C. W.; Hu, X. Nat. Commun. 2016, 7, 12494.

(k) Blaser, H.-U.; Steiner, H.; Studer, M. ChemCatChem 2009, 1, 210.

[6] (a) Gao, Y.; Ma, D.; Wang, C.; Guan, J.; Bao, X. Chem. Commun. 2011, 47, 2432.

(b) Giomi, D.; Alfini, R.; Brandi, A. Tetrahedron 2011, 67, 167

(c) Li, B.; Xu, Z. J. Am. Chem. Soc. 2009, 131, 16380.

(d) Orlandi, M.; Benaglia, M.; Tosi, F.; Annunziata, R.; Cozzi, F. J. Org. Chem. 2016, 81, 3037.

(e) Orlandi, M.; Tosi, F.; Bonsignore, M.; Benaglia, M. Org. Lett. 2015, 17, 3941.

(f) Sharma, S.; Kumar, M.; Kumar, V.; Kumar, N. J. Org. Chem. 2014, 79, 9433.

[7] Pirola, M.; Faverio, C.; Orlandi, M.; Benaglia, M. Chem.-Eur. J. 2021, 27, 10247.

[8] Porwal, D.; Oestreich, M. Eur. J. Org. Chem. 2016, 2016, 3307.

[9] Freeman, A. W.; Urvoy, M.; Criswell, M. E. J. Org. Chem. 2005, $70,5014$.

[10] Duan, Z.; Ranjit, S.; Liu, X. Org. Lett. 2010, 12, 2430.

[11] Shi, J.; Wei, W. Chin. J. Org. Chem. 2020, 40, 2170 (in Chinese). (时建伟, 魏伟, 有机化学, 2020,40,2170.)

[12] (a) Peng, S.; Lin, Y.; He, W.-M. Chin. J. Org. Chem. 2020, 40, 541 (in Chinese).

(彭莎，林英武，何卫民，有机化学, 2020, 40, 541.)

(b) Chen, D.; Sun, Y.; Dong, D.; Han, Q.; Wang, Z. Chin. J. Org. Chem. 2020, 40, 4267 (in Chinese).

(陈德茂, 孙媛媛, 董道青, 韩晴晴, 王祖利, 有机化学, 2020, 40, 4267.)

(c) Ge, L.; Chiou, M.-F.; Li, Y.; Bao, H. Green Synth. Catal. 2020, 1,86 .

(d) Cao, D.; Pan, P.; Li, C.-J.; Zeng, H. Green Synth. Catal. 2021, 2, 303.

(e) Wang, P.; Zhao, Q.; Xiao, W.; Chen, J. Green Synth. Catal. 2020, 1,42 .

(f) Tong, S.; Li, K.; Ouyang, X.; Song, R.; Li, J. Green Synth. Catal. 2021, 2, 145.

[13] (a) Wang, Z.; Liu, Q.; Liu, R.; Ji, Z.; Li, Y.; Zhao, X.; Wei, W. Chin. Chem. Lett. 2021 DOI: 10.1016/j.cclet.2021.08.036.

(b) Meng, N.; Lv, Y.; Liu, Q.; Liu, R.; Zhao, X.; Wei, W. Chin. Chem. Lett. 2021, 32, 258 .

(c) Wang, L.; Bao, P.; Liu, W.; Liu, S.; Hu, C.; Yue, H.; Yang, D.; Wei, W. Chin. J. Org. Chem. 2018, 38, 3189 (in Chinese). (王雷雷, 鲍鹏丽, 刘维伟, 刘思肜, 胡昌松, 岳会兰, 杨道山, 魏伟, 有机化学, 2018, 38, 3189.)

(d) Bao, P.; Liu, F.; Lv, Y.; Yue, H.; Li, J.-S.; Wei, W. Org. Chem. Front. 2020, 7, 492.

[14] (a) Jin, W.; Liu, C. Chin. J. Org. Chem. 2021, 41, 2148 (in Chinese).

(金伟伟，刘晨江，有机化学, 2021, 41, 2148.)

(b) Gui, Q.-W.; Teng, F.; Li, Z.-C.; Xiong, Z.-Y.; Jin, X.-F.; Lin, Y.-W.; Cao, Z.; He, W.-M. Chin. Chem. Lett. 2021, 32, 1907.

(c) Sun, K.; Xiao, F.; Yu, B.; He, W.-M. Chin. J. Catal. 2021, 42, 1921. 
[15] Qu, Z.; Chen, X.; Zhong, S.; Deng, G. J.; Huang, H. Org. Lett. 2021, 23, 5349 .

[16] Smolinsky, G.; Feuer, B. I. J. Org. Chem. 1966, 31, 3882.

[17] Zhao, L.; Hu, C.; Cong, X.; Deng, G.; Liu, L. L.; Luo, M.; Zeng, X. J. Am. Chem. Soc. 2021, 143, 1618.

[18] Li, J.; Shi, X.-Y.; Bi, Y.-Y.; Wei, J.-F.; Chen, Z.-G. ACS Catal. 2011, $1,657$.

[19] Wienhofer, G.; Sorribes, I.; Boddien, A.; Westerhaus, F.; Junge, K.; Junge, H.; Llusar, R.; Beller, M. J. Am. Chem. Soc. 2011, 133,
12875.

[20] Yao, W.; Wang, J.; Lou, Y.; Wu, H.; Qi, X.; Yang, J.; Zhong, A. Org. Chem. Front. 2021, 8, 4554.

[21] Formenti, D.; Ferretti, F.; Topf, C.; Surkus, A.-E.; Pohl, M.-M.; Radnik, J.; Schneider, M.; Junge, K.; Beller, M.; Ragaini, F. J. Catal. 2017, 351, 79.

[22] Lu, H.; Geng, Z.; Li, J.; Zou, D.; Wu, Y.; Wu, Y. Org. Lett. 2016, $18,2774$.

(Lu, Y.) 